[82:81'38]:821.133.1(71).09-31 Dickner N.

7.038.6:821.133.1(71).09-31 Dickner N.

https://doi.org/10.18485/asec_sacs.2021.9.ch17

\title{
Carmen Andrei*
}

Université « Dunărea de Jos », Galaţi, Roumanie

\section{VOIX (NON)FICTIONNELLES DANS L'ANCRAGE DE L'HISTOIRE : NIKOLSKI DE NICOLAS DICKNER}

\section{Résumé}

Le discours fictionnel postmoderne continue au début du troisième millénaire la quête de soi et des racines, l'interrogation identitaire personnelle et collective. Nous proposons une double analyse du soi en mouvement et du passé familial (les aïeux) à partir d'un représentant de la génération des " désaxés affectifs », Nicolas Dickner et de son roman primé - Nikolski (2005). Dans ce roman, Je, le narrateur autodiégétique constitue un point zéro dont la vie apparaît en latence. Il se glisse aussi dans une perspective hétérodiégétique pour suivre la trajectoire à la fois géographique et mémorielle de deux autres personnages, l'un métis, l'autre acadien. Montréal, en particulier le Marché Jean-Talon, constitue le foyer où les trois personnages en mouvance intérieure séjournent (ou demeurent) avant de repartir dans un espace qui couvre les Amériques et au-delà. Mais tous trois effectuent surtout une trajectoire intérieure dans la mémoire familiale et collective. Trois chansons illustreront des aspects du rapport au mémoriel et à l'espace, c'est-à-dire Dégénération (1996) du groupe Mes aïeux, Omniprésent (2012) de Damien Robitaille et Les étoiles filantes (2004) des Cowboys fringants.

\section{Mots-clés}

Cartographie identitaire, boussole Nikolski, Dégénérations, espace, mémoire, Histoire.

\footnotetext{
* carmen.andrei@ugal.ro
} 


\section{Pour introduire à la question identitaire de l'espace dans la grille postmoderne}

L'idée-maîtresse de mon article part des thèses de F. Paré et de L. Gauvin, qui tissent des liens entre les communautés minoritaire et diasporale sur le plan de la mémoire collective et sur le plan structurel de l'espace montréalais. Dans les deux cas, ceux-ci, mémoire et espace, se trouvent vacillants, en mouvance. On les décline par le binôme vivre ici $v s$. ailleurs, jadis vs l'instant présent.

À la fin du millénaire, l'être social a toujours des difficultés à ancrer son moi dans la conceptualisation ou dans le train de vie quotidien. Les spécialistes soulignent cet aspect dans les termes suivants : « [Il a] délaissé certains éléments d'identification traditionnels, tels les liens territoriaux et ancestraux pour endosser une identité à caractère plus personnel, une image de soi plurielle, particularisée et en rupture avec l'attachement traditionnel du soi au Même collectif» (Delic et al., 2011: 1).

Les conséquences cette rupture épistémologique sont la perte de la certitude identitaire, l'isolement du soi, le délitement des rapports avec l'autre / les autres qui mène même à sa marginalisation et à celle de l'autre. Incompatibilité avec l'appartenance à un Nous, à un espace social sécurisant. Par voie de conséquence, on se questionne sur l'appartenance à un espace social et culturel qui soit le vecteur de sa construction identitaire (Ibidem : 2). François Paré a maintes fois souligné que, paradoxalement, dans l'esprit fragmentaire du sujet dit post-identitaire, il y a une volonté de rejoindre autrui (Ibidem : 5).

De nombreuses œuvres littéraires et la plupart des études se penchant sur un corpus minoritaire dans une perspective théorique ont une prédilection pour l'espace. Cet intérêt accompagne selon Élise Lepage l'émergence et la mise en place de champs littéraires particuliers aux différentes régions de la francophonie canadienne (de la fin des années 1960 au milieu des années 1980). Dans un premier temps, on s'intéresse à un espace collectif, linguistique, identitaire, imaginaire. Puis, les écrivains et chercheurs s'intéressent à des lieux mieux identifiés, circonscrits (une ville minière, le village, Moncton, Sudbury, Ottawa).

Les études, géocritiques avant la lettre, mettent en relief la singularité de l'auteur ou un imaginaire rattaché à un lieu. Maintenant, toujours selon Élise Lepage, on assiste à un épuisement du topos critique de l'espace. On se tourne de plus en plus vers l'analyse du paysage, ce qui déprend du monde référentiel et accompagne la maturation esthétique des champs littéraires.

Jimmy Thibeault note pour sa part que depuis les années 1980, certains romans de la littérature québécoise proposent une relecture des identités québécoise et canadienne-française par le biais d'une ouverture des frontières identificatoires traditionnelles (géographiques, culturelles, linguistiques, nationales) et une 
exploration de l'espace continental nord-américain (Thibeault 2011 : 281-284). D'où la floraison du roman de la route, du voyage identitaire qui se fait sur une image de la Franco-Amérique. On réinterprète l'image passéiste, colorée par la perte, pour se tourner vers le dynamisme des récits multiples des individus ayant sillonné le continent depuis son origine.

\section{Nicolas Dickner, Nikolski, 2005}

\section{Autour du texte}

Nicolas Dickner (1972-) est un écrivain et traducteur québécois originaire de Rivière-du-Loup. Il détient une maitrise en création littéraire à l'Université Laval (1996). Il a débuté avec $L$ 'encyclopédie du petit cercle (2000), recueil de nouvelles (Prix Jovette-Bernier et le Prix Adrienne-Choquette de la nouvelle). En 2005, il publie Nikolski [abrégé désormais en $N$ pour les citations, suivi du numéro de page], roman traduit dans plusieurs langues et primé d'emblée (Prix des libraires du Québec, Prix littéraire des collégiens, Prix Anne Hébert, Prix Lavinal Printemps des Lecteurs, Prix des libraires- $15^{\mathrm{e}}$ anniversaire). Il continuera avec Tarmac (2009), traduit également, Le romancier portatif (2011) - un recueil de ses chroniques parues dans l'hebdomadaire Voir. Avec Dominique Fortier il écrit Révolutions (2014) et dernièrement, en 2015, il donne Six degrés de liberté (Prix littéraire du Gouverneur général).

Les critiques ont identifié dans ce roman l'inspiration de l'Oulipo, de Perec : La vie mode d'emploi, Un homme qui dort (Porter 2012 : 297 ; voir aussi l'idée des pièces de puzzle $-N, 13$ ), de Borges, Michel Tournier (Le roi des Aulnes), une structure thématique profonde et inversée qui dérive de Mobby Dick de Melville $(N, 161)$, un vrai dialogue interculturel avec certains classiques du genre. L'incipit, flou, précis et entamant la genèse de l'ouverture à la fois, est illustratif dans ce sens : « Mon nom n'a pas d'importance. Tout débute au mois de septembre 1989 vers sept heures du matin. Je dors encore, recroquevillé dans mon sac de couchage, étendu à même le plancher du salon. [...] Par la fenêtre, on entend le rythme monotone des vagues qui déferlent sur les galets » $(N, 11)$.

Dès le début, Dickner pratique le cache-cache et l'équivoque dans un palindrome auto-réflexif, une forme de miroir qui refuse toute révélation d'identité référentielle ou inférentielle aux lecteurs (Porter 2012 : 299). S'inscrivant dans l'imaginaire de l'Amérique française, il serait un Volkwagen Blues (1984) de Jacques Poulin, du XXI ${ }^{\mathrm{e}}$ siècle (Biron 2007 : 560). Une trame qui couvre douze ans (1989-2001), structurée en vignettes à lire aussi indépendamment, Nikolski se situe dans le sillage de ces romans du voyage, étant également une réflexion métafictionnelle. Conscience forte du continent qui passe par l'image de la diaspora acadienne et canadienne-française. Pour les protagonistes, ce 
sera aussi reconstituer leur culture perdue : malgré les échecs initiaux, ils ne s'abandonnent pas à une " nostalgie paralysante » (Porter 2012 : 297), mais se reconfigurent du point de vue identitaire dans une ville-rhizome, Montréal, «hétérogène et hétéroclite, redéfinie elle aussi selon les désirs de ses arrivants » (Gauvin 2010 : 50) ; à ce titre, à mon sens, ce serait une approche intéressante de se pencher sur des voix féminines autochtones - Nelly Arcan (Putain) et migrantes : Ying Chen, Kim Thuy qui analysent Montréal comme ville-rhizome.

Comme structure, le roman est divisé en cinq périodes étalées entre 1989 et 1999, chaque section ayant une longueur inégale. Comme trame, il retrace l'existence de trois jeunes gens : 1. un narrateur anonyme ( $\boldsymbol{J e})$ autodiégétique, centre de conscience du roman, déclenche le récit, intervient en 1994 (dans le chapitre " L'Enfer ». Le Je brosse un portrait de lui, lucide et ironique : «Il faut dire que j'ai eu de la difficulté à établir les liens avec les gens. Je suis, paraît-il, trop renfermé, trop casanier. Aucune de mes rares amantes n'est parvenue à comprendre que je puisse me contenter d'un boulot de bouquiniste. Un jour ou l'autre, elles finissaient toutes par se demander - et, finalement par me demander - pourquoi je ne voulais pas voyager, étudier, faire carrière ou gagner un meilleur salaire. [...] Pour ma part, je souffre d'une carence : je suis un bouquiniste sans histoire, sans trajectoire propre ; ma vie obéit à l'attraction des livres, le faible champ magnétique de mon destin subit la distorsion de ces milliers de destins plus puissants et plus intéressants. », $N, 168$ ) et clôt le livre. Il travaille dans une bouquinerie ; 2 . Noah Riel se place dans une narration hétérodiégétique. C'est le demi-frère du narrateur Je par le père (Jonas Doucet), mais ils ne le savent pas. Étudiant en archéologie (détritus, puis études autochtones) et 3 . Joyce Doucet, double de Je $(N, 265)$ et sa cousine, mais seul le lecteur le sait, employée dans une poissonnerie près du marché Jean-Talon, pirate informatique. Elle se place dans la narration hétérodiégétique. Il convient de souligner la concentration spatiale : Petite Italie, à Montréal, bouquinerie de Je. Je habite dans la banlieue montréalaise pourvue d'une " douteuse poésie " $(N, 12)$, le nomade Noah s'y installe en arrivant de l'Ouest canadien et Joyce y arrive de Tête-à-la-Baleine (Basse-Côte-Nord). Les personnages se croiseront, mais ne se rencontreront jamais véritablement à trois.

\section{Personnages et relations familiales}

Les personnages sont atypiques, qui se laissent guider par le hasard, les contacts. Jimmy Thibeault montre que leurs origines familiales floues et les rapports cachés qui les relient illustrent la nécessité de refonder l'identité. Ainsi leur cartographie identitaire «n'est plus déterminée par des frontières traditionnelles d'appartenances à un lieu, à une culture, à une nation ou à une langue, mais se déclinent plutôt en fonction des expériences de vie et des contacts avec les autres ", elle s'élargit sur tout le continent et réussit à « s'inscrire dans le sillage de 
l'histoire » sans insister sur une fausse identité (Delic et al. $2011: 12$ ). C'est ce qu'affirme, entre autres, le groupe énergique d'inspiration country, les Cowboys fringants, dans la chanson Les étoiles filantes (2004) : cette génération qui vit dans la contemporanéité aliénante (où il faut assumer de façon plénière le rôle d'animal social avec travail, famille, enfants, hypothèque, etc.) doit subir la réalité dans l'espoir d'être heureuse. Les jeunes ne sont que « des étoiles filantes » se remémorant les bons temps d'une enfance insoucieuse.

Dans son analyse du roman selon la grille ricardienne (de la mêmeté et de l'ipséité), J. Desrochers souligne aussi la complexité des liens qui unissent les protagonistes qui vivent dans l'ambiguïté identitaire, ce qui les oblige à agir, à s'émanciper. Donc, se raconter devient synonyme de se (re)trouver, s'inscrire dans la durée (Desrochers 2017 : 169-171).

Élevés dans une famille monoparentale, ils s'émancipent du nid familial à la fin de l'adolescence et s'autodéterminent (den Toonder, $2011: 14$ ) : Noah vivait dans une caravane avec sa mère métisse, Sarah Riel, et un père de passage, Jonas Doucet. Tous deux gardent un contact sporadique avec le père qui a vécu à Nikolski, puis ils se perdent de vue. Dès que Noah quitte sa mère pour étudier à Montréal, il perd contact avec elle : il lui écrit des centaines de lettres $(550, N$, 141) qui reviennent des postes restantes. Pour Je, c'est particulier aussi : sa mère (libraire elle aussi) est morte d'un cancer ; vagabonde dans sa jeunesse, elle a rencontré un marin acadien, un amant, Jonas Doucet avec qui elle a perdu contact (le père absent envoie rarement des cartes postales à écriture indéchiffrable). Le récit commence justement avec la liquidation des biens maternels (le ressac de son passé). Je retrouve une boussole que son père lui avait donnée, et qu'il nomme Nikolski. Quant à Joyce, sa mère a disparu - elle la cherche, trouve une lointaine cousine pirate informatique, Leslie-Lynn, qui s'est fait emprisonner à Chicago. Elle est élevée par son père, mais véritablement par son grand-père qui lui transmet l'héritage de pirates de la famille.

On a donc affaire à un roman d'apprentissage, marqué par la carence de liens familiaux. Les liens de filiation se font par la généalogie familiale, mais aussi par la diaspora francophone. Pour Laurence Porter, ces protagonistes anonymes illustrent trois réactions distinctes au défi de reconstituer une identité canadienne-française moderne au sein d'une nation à prédominance anglo-saxonne : passivité (Je hiberne dans un monde de livres), rébellion (Joyce et sa piraterie cybernétique) et renouveau (Noah renaît grâce à son fils, Porter 2012 : 308).

Vidangeurs de mémoire et au sens propre, des poubelles, des déchets, de bouquins, nos protagonistes fouillent, nous dirions plutôt « recyclent»; Je se cherche dans la bouquinerie, Joyce dans les ordinateurs, quant à Noah, dans l'archéologie, puis la tradition orale. Leurs trajectoires actuelles se croisent et se ratent merveilleusement, le passé familial commun se dit dans des récits enchevêtrés et 
des images disparates. Une atmosphère troublante brossée par mini-fétichismes (au sens valorisant) : les vieux livres (dont le livre sans visage, belle métaphore à laquelle nous reviendrons !), les déchets, la piraterie, les poissons.

\section{L'espace}

«[L]es petites littératures tendent à glorifier l'espace», souligne François Paré et le roman de Dickner étaie pertinemment ce propos. Il y a d'abord l'île et $\boldsymbol{l a}$ mer, la seconde étant considérée par J. den Toonder comme la métaphore essentielle au roman. En effet, «l'espace textuel est saturé de références à la mer » (Desrochers 2017 : 173). Pour Joyce notamment, ses rapports avec l'île et la mer sont viscéraux : la mer est omniprésente à Tête-à-la-Baleine (Basse-CôteNord) et elle connaît bien les îles avoisinantes (transhumance à l'île Mermette, voisine de l'île Providence ; par ailleurs, il y a un lien entre l'île Providence (Basse-Côte-Nord) et l'île Providence, refuge des pirates, dans les Caraïbes). Mais le lien avec la mer et l'île devient sensible : elle se sent prisonnière de sa famille paternelle, et préfère la lignée maternelle : ancêtres pirates acadiens, oncle (Jonas) enfui et cousine pirate informatique. Quand le grand-père meurt, la mer avale sa maison et on y voit la métaphore de la lignée qui disparaît. Elle poursuit malgré tout la lignée flibustière en suivant la voie de Leslie-Lynn Doucet : elle fouille les dépotoirs à la recherche de matériel informatique (pour retrouver sa mère et sa cousine), puis pour pirater des données personnelles afin de les revendre sur internet. Il apparaît donc une autre métaphore, celle de la pêche : paternel-poisson; maternel-matériel informatique-piraterie. À la fin du roman, elle part retrouver sa mère.

Pour Noah, bien qu'elle sillonne les provinces de l'Ouest, la caravane de son enfance le berce de son roulement. Le père va sur la côte Pacifique ; la mère déteste l'eau et les îles. Il a une première trajectoire vers l'eau et île : études à Montréal. La pêche dans le dépotoir : le personnage archéologue Thomas Saint-Laurent clame que tout est déchet, que ce soit les civilisations anciennes et modernes, dès qu'il y a sédentarité ; une deuxième trajectoire vers l'île : fouilles archéologiques pour retracer des déchets nomades à l'île Stevenson (qui jouxte l'île Providence) et une troisième, vers l'île Margarita (dans les Caraïbes) pour suivre son fils Simón, dont la mère Arizna lui refuse la paternité (comme Sarah Riel et mère de Je par rapport à Jonas Doucet). À l'île Margarita, Noah comptait retracer l'histoire des indigènes, les Garifunas. Mais comme les documents historiques ont disparu, Noah utilise la fiction (par ailleurs, durant ses études, il fréquente la bibliothèque universitaire, section « Sciences navales, récits de voyage et serpents de mer » et, en fait, sa véritable vocation n'est pas d'être archéologue, mais conteur, il fabrique des contes fabuleux pour Simón). Il quitte cette île pour Montréal avec son fils (il y a la possibilité que la mère meure). 
C'est ainsi que dans cette reconfiguration identitaire (élever son fils, lui inventer des histoires généalogiques mises en abyme), il a gagné de l'autonomisation.

Pour Je, la mer est une métaphore qui ouvre le roman : fusion bruit de la mer-ramassage des ordures. Par la boussole, il nourrit le rêve de plages et d'océans. Avec Joyce, il cherche sa boussole tombée dans le sous-sol de l'immeuble, inondé. Cette rencontre l'amènera à briser sa sédentarité et à partir à l'aventure : après les voyages dans le livresque, l'imaginaire, il entamera ceux dans la réalité. En somme, la mer favorise l'émancipation des personnages (cf. Sophie Beaulé, Notes de cours inédites ).

En ce qui concerne un autre espace important, la maison, les études ponctuelles sur Nikolski ont souligné la vision complexe de la maison que donne Dickner : la maison est une carte mentale « multi-sensorielle et multi-scalaire », maison qui «n'est pas un vestige d'une solide modernité, mais [...] une identité ancrée dans une ère de mobilité globale définie de plus en plus par la liquidité et le changement », une sorte de nid pour échapper à la modernité, un endroit de stabilité (Bida 2014 : 34).

\section{La boussole Nikolski : chercher le Nord / le Sud?}

La Franco-Amérique a une image pluraliste, polyphonique. Le roman de Dickner participe à la reconfiguration de l'espace franco-américain par la cartographie d'une appartenance multiple, selon Thibeault, celle des protagonistes au territoire (Thibault 2011 : 287). Cette reconfiguration s'appuie sur la capacité des personnages à créer leur propre histoire et à justifier leur appartenance au continent, ce qui s'effectue par l'abandon du cadre identitaire familial. On a donc une cartographie de la Franco-Amérique, par le lien aux Métis (et Louis Riel), aux Acadiens et à Montréal comme zone de transit. L'identité des personnages s'ouvre sur un espace continental plutôt que régional. Ceci rappelle Waddell et Louden pour qui la franco-américanité est paradoxale : pluralité de foyers, fondée sur un rapport de plus en plus individualiste au monde, réalité de dimension continentale.

La boussole ou « le compas Nikolski » est le point-capiton au sens lacanien du roman. Ce compas de marine a la forme d'une sphère en plastique remplie d'un liquide clair, dans laquelle une seconde, aimantée, flotte. Comme le gyroscope de Nestor dans Le Roi des Aulnes de Michel Tournier, le tout doit assurer la stabilité de la couche interne dans les conditions les plus orageuses. Je la porte comme talisman autour du cou, c'est une fixation, il est hanté par l'idée de la perdre (Porter $2012: 306$ ).

La boussole met en abyme cette rupture : elle n'indique pas le nord, mais Nikolski, dans les îles aléoutiennes (où le père de Je repose dans une tombe 
anonyme) - une déviation qui pointe le parent absent, bien sûr, mais aussi la nécessité pour les personnages de « retrouver leur nord », de dévier de leur trajectoire initiale. Noah quitte l'Ouest pour l'Est (Montréal) puis le Nord et le Sud (Vénézuéla) : passage d'une identité « entre-deux » au retour vers les origines autochtones, Joyce (et sa cousine) quitte le Nord pour le Sud (Montréal, Chicago) et renoue avec l'acadianité, Je quitte la banlieue pour Montréal, puis l'inconnu. Le Québec doit s'ouvrir au continent.

La chanson de Damien Robitaille (chanteur ontarien issu d'une famille exogame linguistiquement), Omniprésent (2012) étaie nos propos. Citons quelques paroles parlantes dans ce sens : " Je suis partout, mais je n'suis pas là », « J'apparais simultanément / Dans plusieurs lieux, et plusieurs événements / Comme un homme omniprésent. De tout bord, de tous côtés / Parfaitement éparpillé / À l'est, à l'ouest, au sud, au nord / Fondu dans tous les décors », « Je suis assez assidu / Il a le don d'ubiquité / À 110\% dispersé. [...] J'ai peur que ma vie dérape / Je suis trop souvent dans les vapes ». Parodie des clips de James Bond, cette chanson brode le thème de l' « ubiquité » (adaptation) qui rime avec liberté et perte d'identité à la fois, en reflétant la réalité de jeunes. Dans La distance habitée, François Paré a réfléchi sur la condition minoritaire. Par exemple, il dégage certaines caractéristiques dans les cultures minoritaires : 1) le désir d'affirmation s'accompagne d'une conscience profonde de la perte ; 2) des stratégies de résistance à la culture dominante ; 3 ) des stratégies d'accommodement. Le sujet minoritaire « habite» au sens fort la distance qui le caractérise : il prend possession des facettes de sa situation linguistique, culturelle. On a dans $\mathrm{Om}$ niprésent de l'hybridité, de la parodie, de la lucidité (Paré 2005).

Nous affirmons que les jeunes héros postmodernes sont désemparés : ils vivent quasiment tous dans la précarité et l'inconfort, la surconscience linguistique. Dans les cas de Je et Joyce c'est tellement ambigu, ils cherchent obstinément à trouver le(ur) Nord. Pour Jonas, ce sera chercher et trouver (?) le Sud. Somme toute, l'existence de cette boussole spéciale apparaît pour insister sur la nécessité de s'orienter, de trouver la « bonne» route.

La diaspora acadienne est maintes fois invoquée : les trois personnages sont liés à la famille acadienne, les Doucet, qui a émigré à Tête-à-la-Baleine avant la déportation de 1755. C'est une appartenance révélatrice, car le peuple acadien se définit par le fait qu'il ne possède pas de territoire identitaire à lui. À cela s'ajoute l'ascendance métisse de Noah : errance des Métis qui ont perdu leurs droits territoriaux et, au-delà, le nomadisme autochtone (dans ce sens, Nikolski est un roman des errances : la mère de Noah, Sarah Riel - dans l'Ouest; la mère de Je fait partie de la beat generation, a traversé le continent ; Jonas Doucet erre sur la côte ouest, après sur l'Océan Atlantique, la cousine de Joyce vit dans l'errance informatique, trajectoire américaine). Cela explique l'urgence que les personnages ont de se positionner dans le monde et de reconfigurer la mémoire 
identitaire à laquelle ils tentent de se rattacher. Noah retrouve son ascendance autochtone en étudiant l'archéologie ; sa rencontre avec Arizna ouvre sa perception des autochtones sur l'espace continental nord et sud-américain. L'échange suivant, entre Noah et Arizna est illustratif dans ce sens :

- Attends un peu... Avant l'arrivée des Blancs, les Inuits se déplaçaient en fonction du gibier et des saisons. Les villages actuels se sont à peu près tous agglutinés autour des comptoirs de la Compagnie de la baie d'Hudson. Ce qui fait qu'au moment de leur relocalisation, les familles d'Inukjuak étaient sédentarisées depuis à peine deux ou trois générations. Alors est-ce qu'on peut encore parler de territoire traditionnel lorsque la manière d'occuper le territoire n'a plus rien de traditionnel ? [Noah]

- Bien sûr ! Le territoire ne se mesure pas en kilomètres carrés. Tu dois aussi considérer les ancêtres, la postérité, la tradition orale, les pistes de ski-doo, les liens familiaux, la chasse au phoque et la pêche au saumon, le lichen, le recours juridique contre Hydro-Québec. Le territoire, c'est surtout l'identité. [Arizna]

Joyce veut devenir pirate comme Leslie-Lynn et les Doucet. Un bel exemple de franco-américanité et la réflexion sur la langue qui suit, la graphie du patronyme montrant les variations selon la génération (les branches généalogiques) et la province (les parcours continentaux). Grand conteur (il fait des récits fabuleux de la famille Doucet, pirates de père en fils), Lizandre Doucet dévoile le grand secret de la famille - « elle était l'ultime descendante d'une longue lignée de pirates dont les tout premiers membres connus s'appelaient Alonzo et Herménégilde Doucette - quoique, selon les circonstances, les lieux ou les subtilités de la grammaire ambiante, on les nommait aussi Doucet, Doucett, Douchette, Douchet, Douchez, Douçoit, Duchette, Ducette, Dowcett, Dusset, Ducit ou Dousette » $(N, 56)$. Par la métaphore du « livre à trois têtes », Dickner suggère ainsi un avenir rédempteur : réparer la diaspora acadienne (Porter 2012 : 309).

Il convient de mentionner également le rôle-clé des cartes maritimes et routières :

Noah se construit par elles un espace identitaire qui ne repose pas sur l'histoire des lieux, mais la sonorité toponymique. Le lien entre le livre sans visage, la carte et l'histoire est récurrent $(N, 38)$. Mais la toponymie évoque aussi de l'imaginaire, pour lui qui cherche à s'enraciner dans le réel. Pour Joyce les cartes représentent un espace de liberté. Pour Aleksandra Bida, cette carte du livre sans visage est « mentale, multi-scalaire, multi-sensorielle et dynamique », son cadre étant « capable d'intégrer les «racines» et les «itinéraires» du constructeur », offre une " cartographie immersive, virtuelle, à quatre dimen- 
sions ", malléable, « une matrice de mémoires sensorielles et paysagères ». (Bida $2014: 7$ )

Montréal joue d'une importance à part. C'est un point de rencontre de différentes cultures (cf. le quartier Petite-Italie). Montréal est le point transitoire de rencontre entre un axe syntagmatique et spatial et un axe paradigmatique correspondant à la mémoire familiale et collective. Elle est un tremplin vers une réinvention du soi ainsi que du collectif. Selon L. Gauvin, la presqu'île de Montréal est un carrefour « susceptible d'accueillir les possibles de tous les destins » (Gauvin 2010:50 - par ailleurs, l'auteure y fait une incursion dans les romans des années ' 60 -' 70 où Montréal est la ville des autres, inhumaine $v s$. la campagne idéalisée, une ville- personnage, réappropriée, des années ' 80 où Montréal apparaît comme la ville-chaos, la ville-fragment), lieu d'(in) achèvement : Joyce et Je partiront, Noah y retourne et y reste.

C'est éminemment le lieu d'affirmation identitaire : émancipation et lien avec l'histoire familiale (poissonnerie et dépotoirs pour Joyce, piraterie féminine comme transgression des traditions familiales; études archéologiques et « réfugié politique », rencontre avec Arizna, qui change la perception des autochtones pour Noah ; lieu de rencontre pour Je). C'est la porte d'entrée du continent : lieu d'arrivée (Noah, Joyce), lieu de départ (Je, Joyce, Noah) et de retour (Noah). On conclut avec L. Gauvin que "Montréal est la ville du presque. Presque cosmopolite, presque française, presque Amérique », « une ville en projet », «Babel moderne écartelée »- linguistiquement et spatialement (verticalité et horizontalité spatiales se conjuguent avec les courants souterrains, Gauvin $2010: 51)$.

\section{Réflexion sur le livre et la littérature}

Nombre de livres et de documents divers (cartes routières et maritimes, lettres, cartes postales, guides de voyage, articles de journaux) montrent la valeur de l'écrit (den Toonder, $2011: 24$ ).

La mise en abyme est évidente : le « livre sans visage » est la métaphore du méta-livre qui contient en fait trois ouvrages anciens (récit de voyage, piraterie) différents et incomplets, la métaphore de ces trois personnages solitaires (il les réunit : Noah a reçu ce livre de son père Doucet, Arizna oublie dans la bouquinerie de Je l'ouvrage qu'elle a emprunté à Noah, Je retrouve le livre plus tard, en compagnie de Joyce). De plus, les personnages sont liés par le sang, comme les trois livres par la reliure. Ce livre tricéphale représente aussi la liberté et l'indépendance des personnages : il n'a pas de propriétaire permanent, est en grande migration : il a été et reste errant éternel, toujours miraculeusement préservé. Une fois volé de la bibliothèque universitaire à Liverpool, il a été avalé par une baleine et vomi de nouveau, remporté par Jonas Doucet lors d'une partie 
de poker à Tel Aviv, transmis à Noah, volé par Arizna, puis abandonné par elle dans la librairie, presqu'inondée, tombé dans le sous-sol inondé du greffier et sauvé là par hasard.

Il représente aussi la liberté que procure la littérature (cf. la bouquinerie de Je). Il contient aussi une métaphore ironique de l'écrivain et, finalement, c'est la métaphore des trois espaces-personnages-provinces : Ouest, Basse-Côte-Nord/ Acadie, Québec/bouquinerie. La métaphore du livre sans visage induit peutêtre l'idée d'une identité franco-canadienne et continentale invisible, "sans visage », à réunir, reconnaître. Son côté passéiste doit être liquidé pour que la francophonie soit ancrée dans l'ici et l'avenir. L. Porter argumente comme suit : « Le Livre en fin de récit renforce peut-être l'idée d'une réunion symbolique des cultures amérindienne, franco-canadienne et latino-américaine, incarnée par Simón, son fils, à l'héritage génétique duquel il ajoute l'élément acadien » (Porter 2012 : 310). Finalement, ce serait la métaphore des espaces autres, rêvés ou interpelés : les États-Unis ou les îles.

À la fin du livre, la carte qui manquait au livre est restituée. Or si le livre retrouve son intégrité (sauf la couverture), il garde encore son mystère - comme le destin des personnages. Nikolski demeure ouvert : la bouquinerie ferme, le livre est mis en « liquidation »-donc le livre est nécessaire au voyage de la vie, et il nous y pousse. Il en va de même pour la boussole Nikolski du narrateur, qui est perdue.

\section{Conclusions}

Nous finissons cette analyse de la quête identitaire non sans insister sur un espace spécial : la bouquinerie située sur la rue Saint-Laurent. C'est le seul endroit qui réunit les 3 personnages (et le fils de Noah), qui ont perdu le contact tangible avec leur passé. C'est un espace comparé à un dépotoir, à un site archéologique ou à une chasse au trésor. Le classement des livres, erratique et impossible, nécessite une grande mémoire, donc c'est un espace multi-temporel et multi-spatial. Le personnage Je s'y dissout, de même qu'il narre les trajectoires de Noah et de Joyce - même si Je ne connait pas les autres. Les deux modes de narration, le récit du soi (autodiégétique) et le récit des autres (hétérodiégétique) s'enchevêtrent suivant la poétique postmoderne, une stratégie narrative qui transforme les « micro-récits » en un «nouveau grand récit» (Thibeault $2011: 317$ ). De fait, l'ensemble du récit que fait le narrateur est un espace de questionnement identitaire du soi. On fait le récit des autres pour trouver sa voie(x). Lorsque Je rencontre les autres personnages, c'est qu'ils ont trouvé leur voie : la boussole est perdue et le livre sans visage est complété.

Notre étude ponctuelle a mis l'accent sur les trajectoires des trois solitudes qui reconfigurent leur cartographie identitaire dans un espace ouvert comme 
Montréal (en particulier le Marché Jean-Talon, dans le quartier cosmopolite de la Petite-Italie) comme point d'arrivée et de départ. L'espace montréalais s'est avéré central sur le plan structurel, mais non pas sur le plan thématique Dans la quête identitaire de ces trois plantes avec et sans racines à la fois, rhizomateux plutôt, le travail de la mémoire et de l'histoire familiale a été décisif sans être pour autant un " agent de déterminisme » (Desrochers, $2017: 173$; l'auteur y développe l'idée de signaux olfactifs qui forme le « smellcape » (paysage olfactif) des protagonistes). Pour Joyce par exemple, les origines ancestrales sont retrouvées grâce à la mémoire sensorielle qui s'active olfactivement dès son arrivée à Montréal à la vue et à l'odorat du poisson.

Nous invitons à ouvrir ce questionnement identitaire dans l'espace et la mémoire en étudiant d'autres trajectoires individuelles et collectives dans d'autres espaces. Il serait intéressant d'analyser d'autres romans qui en parlent tels que : Vortex (2003) de Jean Babineau, L'année la plus longue (2015) de Daniel Grenier, Un vent prodigue (2011) de Simone Chaput. À la question épineuse « Homologuer l'histoire suffit-il pour le besoin ontologique de tout individu de concevoir sa représentation correcte dans la mémoire collective ? », nous osons répondre, toujours dans le sillage des études de François Paré, que la mémoire collective s'abolit souvent dans l'espace et dans le temps et fait éclater les structures stables et fertiles de la reconfiguration d'une Weltanschauung (Paré 2005 : 117). On reste dans un monde diasporal, où la transformation de la mémoire collective au contact de la mémoire collective dominante est manifestement une donnée admise à l'unanimité. François Paré propose de restaurer, il reste à nuancer ce terme dans une future étude, une pensée plus stellaire, plus diasporale » (ibidem : 118). Pour étayer ce propos, on reconfirme que l'histoire personnelle s'homologue après une longue remontée dans le passé familial et collectif(Jonas, le métis - il remonte dans le passé autochtone familial, puis de la nation autochtone, puis des Inuits, puis des Autochtones des Amériques). Pour Joyce, c'est la diaspora acadienne panaméricaine. La revisite de l'Histoire se fera sous un verre grossissant qui dévoilerait dans un premier temps le reniement, le déni d'une certaine appartenance à une communauté (d'où l'exode, la migration), soit la fierté de véhiculer les valeurs des aïeux.

La chanson du groupe du courant néo-traditionnaliste Mes aïeux, Dégénérations (2004) renferme ce questionnement : on y parle de quatre générations d'hommes et de femmes qui ont évolué de manière différente : les arrière-arrière-grands-parents, défricheurs vivant dans la grosse misère ont légué leurs propriétés à leurs enfants, géniteurs de marmaille qui, à leur tour, sont devenus millionnaires (les grands-parents), les parents - génération yé-yé, émancipée $\mathrm{y}$ compris sexuellement - lèguent à leurs fils qui vivent dans la jeunesse insouciante, de l'argent et de bons postes au ministère. Les aïeux et leur mémoire collective fonctionnent donc comme des rhizomes. 


\section{CORPUS}

Dickner, Nicolas. Nikolski. Montréal : Alto, 2005.

Robitaille, Damien. Omniprésent, 2012, disponible sur https://www.youtube.com/ watch? $\mathrm{v}=$ gejgbJqawVw.

Mes aïeux, Dégénérations, 2004, disponibles sur https://www.youtube.com/wat$\mathrm{ch} ? \mathrm{v}=\mathrm{k} 2 \mathrm{~V} 3 \mathrm{NrvThlg}$.

Cowboys fringants, Les étoiles filantes, 2004, disponible sur https://www.youtube. $\mathrm{com} /$ watch? $\mathrm{v}=\mathrm{xjOaT3} 3 \mathrm{bzfY}$

\section{BIBLIOGRAPHIE}

Biron, Michel, Dumont, François et Nardout-Lafarge, Élisabeth. Histoire de la littérature québécoise. Montréal : Boréal, 2007.

Bida, Aleksandra. «The „,complex map” of Home in Liquid Modernity : Re-thinking Mobility and Stability in Nicolas Dickner's Nikolski », in : Canadian Literature. Vancouver : Iss. 222 / Autumn 2014, 34-50.

Delic, Emir, Hotte, Lucie et Thibeault, Jimmy. « Devenir soi avec les autres. Identité et altérité dans les littératures francophones du Canada », in : Analyses, vol. $6, n^{\circ} 1 / 2011,1-14$.

Desrochers, Julien. « Origines et reconfigurations identitaires dans Nikolski de Nicolas Dickner », in: Transmissions et transgressions dans les littératures de l'Amérique francophone (dir. Cecilia W. Francis et Robert Viau). Moncton : Éd. Perce-Neige, coll. « Archipel / APLAQA », 2017, 169-183.

Dumitriu-Panaitescu, Corina (dir.). Dicţionar de francofonie canadiană [Dictionnaire de francophonie canadienne]. Iaşi, Ed. Universităţii « Al.I.Cuza », 2011.

Gauvin, Lise. « Comment peut-on être Montréalais » : fiction d'une ville », in : Managing Diversity and Social Cohesion: the Canadian Experience (éd. Diana Yankova). Association d'Études Canadiennes en Europe Centrale, Masaryk University Press, Brno : 2010, 41-53.

Lepage, Élise. « Cadrages contemporains sur les paysages des littératures francophones du Canada ", in: Temps zéro. Écritures contemporaines Poétiques, esthétiques, imaginaires, $\mathrm{n}^{\circ} .10 / 2015$, disponible sur http://tempszero. contemporain.info/document1308

Paré, François. Les Littératures de l'exiguïté. Hearst : Éditions Le Nordir, 1992.

Paré, François. La distance habitée. Sulbury : Éd. Presse de la parole, 2005. 
Porter, Laurence M. « Mending the Acadian Diaspora : Strategies of Remediation in Nicolas Dickner's Nikolski (2005) », in : University of Toronto Quaterly, vol. $81, \mathrm{n}^{\circ} 2 / 2012,297-312$.

Thibeault, Jimmy. « Reconfigurer la cartographie de la Franco-Amérique : la représentation de l'espace et du récit identitaire dans Nikolksi de Nicolas Dickner », in Analyses, 6, n $1 / 2011$.

Toonder, Jeanette den. « Lieux de rencontre et de transition : espaces liminaires et zones de contact dans Nikolski », in Francophonies d'Amérique, n³1/2011, $13-29$.

Vaillancourt, Claude. Anthologie de la littérature québécoise. Montréal : Éd. Beauchemin, coll. « Chenelière Éducation », $3^{\mathrm{e}}$ édition, 2018.

Coda : Nous tenons à remercier infiniment Sophie Beaulé, professeur à Saint-Mary's University, Halifax, Nouvelle Écosse, Canada pour les pistes d'analyse suggérées, les articles ponctuels sur Nikolski et toutes « les bonnes ondes » envoyées. 